\title{
Formal and Psychological Aspects of Modern Business Notations
}

\author{
V.G. Kalashnikov ${ }^{1, *}$, G.R. Gabidullina ${ }^{2}$, S.M. Mukhametshin ${ }^{3}$, A.S. Galimova ${ }^{2}$, and A.M. \\ Ableeva $^{4}$ \\ ${ }^{1}$ Sterlitamak Branch of Bashkir State University, 453103 Sterlitamak, Russia \\ ${ }^{2}$ Bashkir State University, 450076 Ufa, Russia \\ ${ }^{3} \mathrm{RN}-\mathrm{BashNipiNeft}$ \\ ${ }^{4}$ Bashkir State Agrarian University, 450001 Ufa, Russia
}

\begin{abstract}
The article discusses the features of the transition to the sixth technological order based on the digitalization of the economy, informatization and computerization of all spheres of human life. The conclusion is made about the influence of the ongoing phase shift of the social order on the social and psychological features of modern man. In particular, the increasing role of visual culture and visual (visual-logical) thinking and the corresponding graphic language is noted. The advantages of such a graphic language in terms of information capacity are noted. There is a growing interest in visual forms of modeling production systems in the form of business notations for the purpose of analyzing business processes for their modeling and controlling. The essence and features of the business notation system in various versions are considered. The possibility of combining different business notation systems as different ways of describing a managed object is emphasized. The article focuses on the use of business notation as a means of developing managerial thinking of managers as a multidimensional and contextual visual-logical process that meets the requirements of the modern information environment.
\end{abstract}

\section{Introduction}

At present, there is an obvious phase crisis that marks the transition to a new way of life, which is called the "sixth technological", affecting not only the sphere of production, but also all other spheres of human and human life. S.Y. Glazyev points out that the technological way of life is a production system that involves a closed cycle of technologically coupled productions [6]. They implementation of the cycle includes the stages from receipt of raw materials to finished products in accordance with the type of public consumption. At the same time, the main forms of production are the core, and technological innovations are the key factor in this technological structure, which ensure the evolution of core technologies within the framework of supporting industries. The mode of production of consumer goods determines the entire lifestyle of people, as well as

\footnotetext{
${ }^{*}$ Corresponding author: transmeta1@yandex.ru
} 
various communities - from the family to society. This is why it is so important to study the impact that technological innovations have on both production processes and social relations, as well as on the psyche of people.

\section{Materials and methods}

The key factor of the sixth technological order that is being formed today is the informatization of all aspects of human life, which became possible due to the appearance of computers and the formation of a global computer multimedia network. All other features that researchers attribute to the characteristics of the sixth technical structure are derived precisely from the use of computer systems and their complexes. Thanks to computerization, advances in nano-technologies and additive technologies, as well as cognitive technologies, have become possible. Moreover, the well-publicized convergence of nano-, bio-, info -, and cogno-technologies (NBICS-convergence, eng. NBIC) has become possible thanks to a single platform on which all research in these areas is developed. Modeling and manipulation of micro-and nano-objects, including biological objects, modeling of cognitive processes in the form of artificial intelligence and its increasing use - all this is a product of computer technologies. In the economic sphere, this leads to the emergence of the phenomenon of the "digital economy," which is a management system based on information and computer digital technologies with the corresponding transformation of the production cycle. and the labor market $[5 ; 18]$.

\section{Results and discussion}

An increase in the intensity of the information flow, an increase in the volume of human interaction with computer algorithmic systems lead to certain psychological effects. Among them not only the formation of a "clip mentality" by reducing the time of engagement, and a decrease in perceived fragments of information and difficulty establishing logical links between them, but also a general shift to visual and procedural perception and thinking. This means that the thinking of modern people is becoming more and more focused on the perception of information not in the form of large texts, but in a visual form - in the form of visual presentations or short videos. All this allows us to talk about a specific-visual-culture and the corresponding field of scientific and practical research-visual research (H. Belting, A.V. Venkova, G. BEM, J. Berger, A. Yu. Zenokova, I. N. Inishev, P. Claudel, N. Mirzoev, T. Mitchell, K. Moxey, E. V. Petrovskaya, etc.).

Moreover, some researchers talk about the formation of the next stage of thinking, which is replacing verbal and logical thinking (which until recently was considered the pinnacle of human conscious thinking). This is visual-logical thinking, which operates as units not with words and sentences, but with signs and multi-valued symbols. Since the operations of logical thinking are well studied in psychology (M. Wertheimer, L. S. Vygotsky, P. Ya. Galperin, V. V. Davydov, J. Piaget, Ya. A. Ponomarev, S. L. Rubinstein, O. K. Tikhomirov, B. D. Elkonin, and many others), it becomes clear that thinking based on visual logic should also have its own forms of analysis and synthesis, abstraction and concretization, comparison and generalization, as well as making judgments and conclusions on this basis. The advantages of visual-logical thinking are recognized by many researchers and practitioners: Wilber, D. Roem, V. P. Zinchenko, V. M. Munipov, V. M. Gordon, V. I. Zhukovsky, D. V. Pivovarov, R. Yu.Rakhmatullin, G. P. Shchedrovitsky). In particular, Russian researchers have argued that "visual thinking is a kind of dialectical negation of the negation of actual practice, has both the advantages of universality (as well as verbal thinking), and the property of direct reflection of reality" [7, p.13]. In turn, K. 
Wilber also considers visual (visual-logical) thinking to be the highest level in comparison with abstract-logical (sign and verbal) [13].

The practical developments of O. G. Bakhtiyarov's psychonetics, which includes specific visual psychonetic languages [3], are based on a similar understanding, which makes it possible to collapse and expand significant amounts of information using graphic signs. In addition, a similar logic lay in the basis of the didactic developments of domestic teachers - V. F. Shatalov's reference signals (notes) [15] and V. E. Steinberg's didactic multidimensional tools [16], which, according to the rigor of their construction, exceed the well-known mental maps of T. Buzen [4]. Visual thinking has also found application in business (D. Roem) [12], including in the form of drawing up mental maps (H. Muller) [11].

In the field of management, compliance with these trends in the predominance of visual information over text is most clearly manifested in the creation of a business notation system, which acts as a graphical language for modeling business processes (Business Process Model and Notation, BPMN). A business notation system is a formalized recording method that allows you to design and analyze business processes, as well as analyze various management situations. These notations are used for modeling and controlling business processes. A model of a managed business system created using a particular business notation can be displayed in a variety of software tools for modeling business processes-MS Visio, ARIS, Archi, etc. It can be argued that the use of such a tool for displaying management information not only serves as a means of expressing existing ideas, but also, in turn, has a significant impact on the formation of a specific type of system-algorithmic thinking of the manager using this tool.

This is due to the fact that visual-logical thinking involves an attempt at a dialectical synthesis of abstract sign thinking and the visibility of visual images. It is known from history that writing was initially formed as a language of pictograms (a visual example of which is the Egyptian hieroglyphs or the Mayan language), which then acquired a different form: more visual in some cultures (hieroglyphs of Southeast Asian cultures) and more abstract in other cultures (Phoenician writing, which gave the basis for all varieties of European writing). At the same time, as shown by numerous studies (A. I. Kobzev, R. Levin, J. G. Miller, R. E. Naisbit, A.V. Smirnov, E. Sepir, B. L. Wharf, R. A. Shveder, R. T. Ames, etc.), the form of writing significantly affects the mentality of native speakers of this language and writing. Accordingly, the evolution of the human psyche and, in particular, the processes of information processing (attention, perception, memory, thinking) in the information and computer age requires adequate means of fixing neoplasms, as well as increasing the degree of management of cognitive processes.

On the one hand, visual (graphic) language is a tool for such expression of visual thinking, and on the other hand, a tool for its formation and management. For the first time graphic schemes for describing dynamic events were used during the Russian-Turkish war in 1877 by the polygraphist I. D. Sytin in an Appendix published by him ("For newspaper readers. Manual") supplemented verbal newspaper descriptions of military operations with visual diagrams-drawings on maps consisting of blocks (fortifications and military units) and arrows (their movements). In the future, this language of graphic schemes throughout the first half of the 20th century was used mainly by the military. However, since the second half of the last century, the situation began to change due to the rapid growth of information flows in the management of engineering and design developments, production processes and military actions, accompanied by the need to use more concise than traditional verbal methods of managing management information.

Attempts to create such a graphic language were made especially intensively in the USSR within the framework of the Moscow Methodological Circle (MMK), the founder and permanent leader of which was G. P. Shchedrovitsky. V. A. Lefevre and G. P. 
Shchedrovitsky. Starting in 1975, they developed the graphic "language of schematic images" (YASI) [17], which was further developed in the works of O. S. Anisimov and the Moscow methodological and pedagogical circle founded by him [2]. This system of graphical notation was created as a tool for methodological analysis of any phenomena in the course of collective mental activity. Accordingly, it was used for a concise representation of mental models of various types of problems - from philosophical to applied-in the field of organization of knowledge, training, production and management.

From the very beginning, this language of schematic images began to exist and develop in several varieties due to the creators ' dissimilar understanding of the purpose of this graphic language: materialization of ideal objects of thinking (V. A. Lefevre), a means of organizing meanings in the process of communication (G. P. Shchedrovitsky), the process of thinking as a process of operating with signs (O. S. Anisimov), reflection of the action of the "logical machine as an algorithm for generating new knowledge (V. S.-B. Byazyrov) [8; 10]. It is obvious that its use influenced the thinking style of the analysts who used it-from V. I. Lefevre, G. I. Shchedrovitsky, O. I. Genisaretsky to V. A. Nikitin, P. G. Shchedrovitsky, S. B. Pereslegin.

It is interesting to note that despite the existence of the "iron curtain," many processes in the West and in the USSR went on in parallel, although almost independently. The same can be said about creating a graphical language used for recording business processes. For example, modern business notation systems were developed by the U.S. military around the same time that the Soviet Union developed methodological YASI, which was later used, among other things, for modeling design and production processes.

It should be noted that around the same time, the Soviet Union developed its own systems for managing production and design processes, as well as corresponding graphic descriptions and even three-dimensional models (for example, in the SCALAR system). The need to develop such methods and methods for visualizing processes was explained by the fact that the Soviet system of planned management in the 1960s faced a limit of complexity that created a lag in labor productivity from Western companies - namely, in the field of management organization. These works are primarily associated with the names of two theorists and practitioners of complex systems management: P. G. Kuznetsov and S. P. Nikanorov.

P. G. Kuznetsov headed the Laboratory of Systems Development Management Systems (Lasurs) that existed in 1967-1970. In 1962, S. P. Nikanorov proposed the concept of network management planning (SPM), and soon began working with P. G. Kuznetsov on conceptual management systems. In particular, they developed the SPUTNIK complex (network Planning and Management Systems for Technical Research Teams), which was a sequence of procedures necessary to obtain a complete plan and to be able to calculate the critical path. The tabular form for presenting the programs of activities of SCALAR organizations was developed in the late 1960s.

Later, S. P. Nikanorov created a sow-a system of organizational management. In 1969, S. P. Nikanorov put forward the principle of genetic design, which included operating libraries of models (concepts), the combination of which gives the desired solution (design, management) and allows one to save the essence of the organization with various changes in its configuration. Each library model is a formal axiomatic system, and the design process acts as a system of operations on the models of this library for the synthesis of a project concept or management decision. On this theoretical and methodological basis, in the late 1970s, a 40-volume technical project of the computer design system (TSA), consisting of three blocks, was created by order of the State Construction Committee of the USSR. The logic block of the proposed system was intended for manipulating circuits. By combining the schemes and models proposed by the system, the construction manager could find the best way out of difficult situations. 
This concept was not fully implemented, but it largely served as the basis for the "COMPASS" org system used in the early 1980s during the construction of the Kalinin NPP, developed by Nikanorov's followers (V. G. Vodianov and others). This system proposed to consider the organization as a self-developing system that exists on the basis of network planning, collegiality, monitoring the state of each team member and activating the creative potential of all employees.

Unfortunately, due to a number of political and organizational reasons, the described developments were not widely used in the Soviet management system. This, in particular, explains the success of the Soviet lunar program, as well as the success of the United States in the "Lunar race," which the U.S. won largely due to the "configuration management" system created there which ensured the coordinated actions of numerous organizations and enterprises involved in the lunar program. This is due to the fact that already in the 1940s there began to develop intra-organizational activity "Systems and Procedures" (S\&P). In general, the American system of configuration management of production was created in the late 1950 s and early 1960 s, and computers were initially introduced in organizations to make changes to regulations that affect complex and poorly tracked systems of interconnected processes in a variety of heterogeneous organizations [14]. Initially, the recording, analysis and generalization, as well as the transfer of experience as routine descriptions were made in a simple and concise verbal form. However, in the future, fixation and analysis began to occur in an increasing volume in a graphic form.

Due to the lag in the theory of managing complex systems at the turn of the $21^{\text {st }}$ century that had developed by the mid-1980s, the domestic post-Soviet management practice began to borrow foreign (primarily American) developments in this area, including in the field of graphical analysis and design of business processes and structures. Here is a brief overview of the most common business notation systems.

The Flowcharting notation was created in the ANSI standard in 1970 and contains a very simple set of characters. Flowcharts were the forerunners of modern business process modeling notations. This notation does not have a strict standard, which allows you to flexibly model business processes in different aspects, adding objects to the model as needed. However, this lack of rigor does not allow us to give this model the status of an unambiguously readable graphical language for describing business processes, which reduces its ability to be used as a communication tool.

In 1981, one of the departments of the U.S. Air Force developed an industrial automation program under the abbreviation ICAM (Integrated Computer Aided Manufacturing) and a set of standards IDEF (ICAM Definition) for it. One of the standards of this set was the functional modeling notation IDEF0 (Integrated Definition Language). This method of graphical display was intended to provide group work on creating a model of production processes. This model focuses on functions and visually represents a set of blocks ("black boxes") that have inputs and outputs, control actions, and mechanisms for executing processes, and this system is DETA-lyzed (decomposed) to the required level. This notation focuses on the subordination of objects, since it does not express the sequence of works in time, but only their logical relationships (following, preceding, generating, etc.). The IDEF0 notation has changed somewhat over time and has survived several revisions to the IDEF5 level, but it is currently losing popularity because describing and reading business processes is inconvenient and complex, and, most importantly, programs that support IDEF have become obsolete along with it.

The VAD (Value-Added Chain Diagram) notation proposed by M. Porter focuses on modeling business processes that "create value" in the form of services or products for the consumer. The business process model built in VAD notation provides general view of the company's business processes on a single model for strategic management purposes. There are also some extensions of this notation system. For example, the ARIS tool developed by 
the specialists of the German company IDS Scheer AG allows you to show smaller details on the business process model - performers, risks, documents, data, etc. However, in practice, after modeling business processes at the top level in VAD notation, more detailed modeling of business processes in other notations follows.

To develop the methodology of the ARIS Toolkit, A.V. Scheer developed the EPC (Event-Driven Process Chain) notation. In this notation, the business process is modeled as a list of steps triggered by events, including actions (tasks), inputs and outputs (resources), logical conditions for relationships between them, and also allows you to assign responsibility for performing actions to the employee or their group. The notation is useful for subsequent regulation of the business process, as well as for analyzing the information flow of the business process (incoming / outgoing documents). There are many variations of EPC notation, in the format of columns, rows, and with different lists of objects used.

The VSM (Value Stream Mapping) notation was created in Toyota Corporation, where this abbreviation has its own interpretation - "map of material and information flows." This notation was developed as part of the lean production methodology for analyzing the effectiveness of the business process in Lean 6Sigma projects. The value stream map depicts the physical environment and flows of materials and products in production and is used to map the resource and time spent, and thus assess the performance of a given process, as well as its bottlenecks and the localization of excess inventory. VSM notation is aimed at involving all participants in the business process analysis in order to encourage them to independently search for optimization opportunities, and therefore it is not strict enough in terms of the graphical expression tools used.

The SIPOC notation (Supplier, Input, Process, Output, Customer - Supplier, Input, Process, Output, Order-chick / Consumer) is a process documentation template also adopted in the Six Sigma methodology. In fact, this is not an independent model notation, but a table format that allows you to describe the business process at the top level to determine its boundaries, as well as the interacting parties and inputs/outputs of this process. This allows you to structure the selected business process for analysis and optimization. The absence of complex logic for the interaction of multiple objects makes it possible to use this notation as a tool for joint analysis by employees of business divisions, although it reduces the possibility of using it as a full-fledged graphical language.

The BPMN 2.0 notation (Business Process Model and Notation) is created by the Object Management Group (OMG) consortium and is intended for modeling business processes in order to automate them (turning the model into executable code, that is, a computer program). In this notation, in addition to the steps of the business process, you can model the starting, intermediate, and ending events of the process, information flows, and message flows. Organizing the business process in the Swim Lane format ("swimming paths" separating the performers with lines) makes it easy to transfer responsibility and work flow between the participants in the process, but it makes it difficult to model in the case of several co-performers in a single operation. BPMN is probably the most advanced notation with great features, but it is also difficult to learn and use.

A specific tool is the DFD (Data Flow Diagrams) methodology, which is intended exclusively for describing the flow of information between sources, repositories, and recipients. Two notations are used to describe DFD diagrams - Yordana (Yourdon) and Gane-Sarson (Gane-Sarson), but both of these options can only be used as a tool for analyzing and designing information systems that support business processes and are used by the creators of the corresponding software solutions (programmers, not managers).

Recently, the UML (Unified Modeling Language) notation, created By G. Butch, J. Rambo, and A. Jacobsen, has become more widely used. It is a graphical description language for modeling business processes, system design, and displaying organizational structures, including for the development of appropriate software. UML diagrams are 
created using graphical symbols for things (classes, objects, etc.) and relationships. However, diagrams can be either structural (showing the hierarchy of components) or behavioral (functional). Due to its versatility and the ability to generate program code based on its models, UML notation is one of the most popular means of displaying business processes. However, this tool is mainly used by programmers or for communication between managers and programmers during the automation of business processes.

SDL notation (Specification and Description Language) is a language that models the architecture and behavior of distributed systems and was created to describe processes in the telecommunications sector. It is considered as an extension of the UML graphical language to increase the power of the resulting modeling tool by adding features for hierarchical decomposition of processes. Although these notations are primarily used for creating business process software, their use allows the manager to better model system relationships in a managed complex system.

\section{Conclusions}

So, as a brief analysis shows, each business notation has its own area of application and they all complement each other, which is also noted in [1]. For example, organizations use VAD notation to model business processes at the top level, but SIPOC notation is more often used for primary modeling of the business process selected for optimization. To create detailed models of business processes, the BPMN notation is used for modeling cross-functional interaction, or the EPC notation is used for detailed modeling in order to formalize the information flow and the set of objects of a given business process. A whole group of notations - DFD, UML, SDL - is aimed primarily at displaying and algorithmizing business processes, likening them to computer programs that are created as a result of modeling real processes. Thus, the information and computer approach is increasingly formatting business processes, as well as their understanding by managers.

Thus, the analysis allows us to conclude that visual languages contribute to the formation of a new (higher) type of integral thinking in a person, in which the dialectical removal of the contradiction between visual-figurative (concrete) and verbal-logical (sign, abstract) ways of presenting information occurs. In a single visual form, it is possible to "archive" a significant amount of information about the structure, functioning and development of the object under consideration. These patterns also apply to the management method of modeling business processes through a variety of graphical notations. Since different business notations emphasize different properties of the simulated processes, their constant application has a certain impact on the manager's thinking. In particular, the reference to different display systems of the managed system forms a contextual approach to the analysis of management situations for the manager, which implies the purposeful use of different description systems as contexts for building a multifaceted model of a single object [9].

The results of our research allow us to draw another conclusion: the increased use of digital technologies increases the demand for new skills. First, employees in various fields of activity must acquire competencies in the field of ICT (information and communication technologies) in order to be able to use such technologies in their daily work - for example when accessing information on the Internet or using software. Secondly, the production of ICT products and services - software, web pages, e-Commerce, cloud computing and big data - requires appropriate training, which is largely provided by the formation of visual and logical thinking through the use of business notation systems.

This suggests the need for targeted training of future managers in the use of several systems of business notation, which, along with the method of case analysis (analysis of business situations), should become one of the main tools for the formation of specific 
thinking of the spectator-logical type. The use of these tools makes it possible to form not just an algorithm ("computer-like"), but a system-multidimensional (contextual), threedimensional manager's thinking, which is required by the increasing complexity of an increasingly rich information environment of the present and the foreseeable future.

\section{References}

1. A. S. Alenikov, I. V. Mamonova, K. I. Koloeeva, Bulletin of the Academy of knowledge, 39(4), 38 (2020)

2. O. S. Anisimov, Encyclopedia of managerial knowledge, 439 (2005)

3. O. G. Bakhtiyarov, Post-Information technologies, 87 (1997)

4. T. Buzen, B. Buzen, Change your life with the help of intelligence cards: TRANS, 280 (2017)

5. G. R. Gabidullina, A.M. Luzhneva, U. A. Fedenko, Economics and management: a scientific and practical journal, 3(153), 165 (2020)

6. S. Yu. Glazyev, Theory of long-term technical and economic development, 310 (1993)

7. V. I. Zhukovsky, D. V. Pivovarov, R. Yu. Rakhmatullin, Visual thinking in the structure of scientific knowledge, 178 (1988)

8. A. Zinchenko, G. P. Shchedrovitsky, Technologies of schematization in SMD methodology, https://www.fondgp.ru/

9. V. G. Kalashnikov, Reports of Bashkir University, 1(1), 221 (2016)

10. P. Meytuv, V. Butorin, Kentavr, 1, 9 (1994), http://metodologika.ru/

11. H. Muller, Composing mental maps. Method of generating and structuring ideas: TRANS, 128 (2007)

12. D. Roem, Visual thinking. How to "sell" your ideas using visual images, 300 (2013)

13. K. Wilber, Integral psychology: Consciousness, Spirit, Psychology, Therapy, 412 (2004)

14. S. B. Chernyshev, Corporate entrepreneurship: from meaning to ideal, 685 (2001)

15. V. F. Shatalov, Pedagogical search, 159 (1987)

16. V. E. Shteynberg, Didactic multidimensional tools: theory, methodology, practice, 304 (2002)

17. G. P. Shchedrovitsky, Selected works, 800 (1995)

18. Skills for the digital world. Meeting of Ministers in 2016 against the backdrop of the digital economy report. OECD digital economy papers No. 250, https://www.oecd.org/ 